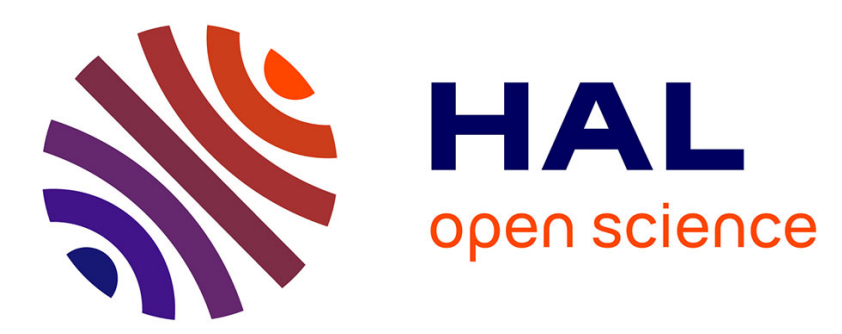

\title{
Effect of a metallic coating on the thermal conductivity of carbon nanofiber-dielectric matrix composites
}

\author{
Jose Ordonez-Miranda, Ronggui Yang
}

\section{To cite this version:}

Jose Ordonez-Miranda, Ronggui Yang. Effect of a metallic coating on the thermal conductivity of carbon nanofiber-dielectric matrix composites. Composites Science and Technology, 2015, pp.7. 10.1016/j.compscitech.2015.01.010 . hal-01134038

\section{HAL Id: hal-01134038 \\ https://hal.science/hal-01134038}

Submitted on 24 Mar 2015

HAL is a multi-disciplinary open access archive for the deposit and dissemination of scientific research documents, whether they are published or not. The documents may come from teaching and research institutions in France or abroad, or from public or private research centers.
L'archive ouverte pluridisciplinaire HAL, est destinée au dépôt et à la diffusion de documents scientifiques de niveau recherche, publiés ou non, émanant des établissements d'enseignement et de recherche français ou étrangers, des laboratoires publics ou privés. 


\section{Effect of a metallic coating on the thermal conductivity of carbon nanofiber-dielectric}

\section{composites}

\section{Jose Ordonez-Miranda, ${ }^{1, *}$ and Ronggui Yang ${ }^{2}$}

${ }^{1}$ Laboratoire d'Énergétique Moléculaire et Macroscopique, Combustion, UPR CNRS 288, Ecole Centrale Paris, Grande Voie des Vignes, 92295 Châtenay Malabry, France.

2 Department of Mechanical Engineering, University of Colorado, Boulder, Colorado 803090427, USA.

\section{Abstract}

A model is developed to evaluate the thermal conductivity of composites made of a dielectric matrix material containing randomly oriented and aligned carbon nanofibers coated with a metallic layer. The effect of the metallic coating on the phonon thermal conductivity of the matrix material and the electron-phonon coupling inside the metallic coating are both taken into account in this model. It is shown that: 1) the metallic coating has an extraordinary effect on the enhancement of the composite thermal conductivity. For a volume fraction of $30 \%$ of fibers with radius of $50 \mathrm{~nm}$ and $10 \mathrm{~nm}$-coating of copper, the thermal conductivity increase is as high as $27 \%$, which increases significantly with the radius of the fibers, their volume fraction, and the thickness of the coating. 2) Although the thermal conductivity of silver is $453 \%$ as that of indium, the composite thermal conductivity is only increased slightly by changing an indium coating to a much more expensive silver coating, due to the relatively high thermal conductivity of these metals in comparison with the one of the matrix. 3) The composite thermal conductivity increases with the volume fraction of the fibers when their radius and radial thermal conductivity are greater than the matrix-coating Kapitza radius and the effective thermal conductivity of the matrix, respectively. The obtained theoretical results match well experimental data reported in 
the literature for the thermal conductivities in the directions parallel and perpendicular to the axis

of aligned carbon fibers. This model is expected to be valid for composites in the absence of percolation with the length-to-radius aspect ratio of fibers in the range of 10 to 100 , and it provides theoretical guides for optimizing cost-efficient high thermal conductivity composites.

Keywords: Composites; Carbon fiber; Metallic coating; Thermal conductivity.

PACS: $61.46 .-\mathrm{w}, 65.80 .+\mathrm{n}, 65.60 .+\mathrm{a}$.

*Corresponding author

Telephone: +33684107141

Email: jose.ordonez@ecp.fr

\section{INTRODUCTION}

Nanostructured carbon materials such as carbon nanotubes (CNTs), graphene and carbon nanofibers (CNFs) are widely used as reinforcement materials for composites because of their outstanding mechanical, thermal and electrical properties, which can significantly improve the performance of materials for industrial applications [1-3]. It is very common to optimize conflicting requirements on the material properties by combining the most useful properties of two or more phases, which does not ordinarily appear together in nature. Especially, carbonfiber-reinforced polymers have become essential nowadays in aerospace and automobile industry. For example, using CNFs instead of steel can lower the weight of the involved components by up to $50 \%$, which can improve fuel economy by as much as $40 \%[2,4]$. Typical aspect ratios (length/diameter) of these fibers are on the order of tens to hundreds, which facilitate the control on their random or aligned distribution inside the matrix, and the understanding and prediction of the physical properties of CNFs-based composites. 
Significant research efforts have been dedicated to improve the thermal conductivity of composites made up of CNFs embedded in a dielectric matrix for thermal management applications [5-7]. The poor thermal conductivity of the CNFs along their radial direction, which can be as low as three orders of magnitude smaller than the one along their axial direction, has limited the improvement. Metallic Coating of the CNFs with a metallic layer has been proposed recently to overcome this problem, with production techniques developed $[8,9]$. Taking into account the relatively high thermal conductivity of metals, the surface metallization of CNFs is expected to enhance the overall thermal conductivity of these composites and therefore extending their applications. However, the thermal performance of these composites is still not well understood. Given that the thickness of the coating layer can be of the order of the mean free path of the energy carriers (electron and phonons), which is typically of a few nanometers for metals at room temperature, the thermal conductivity of these fibers and therefore of the whole composite is expected to depends strongly on interactions of energy carriers and their scattering processes with interfaces [10-15]. Currently there are no theoretical models that can provide design guidelines.

The objective of this work is to develop a theoretical model to quantify the thermal conductivity of composites consisting of a dielectric matrix embedded with randomly oriented CNFs that are coated with a metallic layer. The effect of the metallic coating is determined by comparing the enhancement of the thermal conductivity of composites as a function of coating thickness for different metallic coatings. This work could shed some light on the design of high thermal conductivity CNF-dielectric composites. 


\section{THEORETICAL MODEL}

Figure 1(a) shows a composite consisting of CNFs with a metallic coating, embedded in a dielectric matrix. The effective thermal conductivity of these fibers is anisotropic and it has the values $k_{\|}$and $k_{\perp}$ in the axial and radial directions, respectively. The coating and the matrix are assume to have the isotropic thermal conductivities $k_{c}$ and $k_{m}$, respectively. Starting with the our previous thermal conductivity model valid in the dilute limit of particles (Section II.A) [11], the theoretical model for the composite thermal conductivity is developed here, by taking into account the fiber-fiber interaction at the non-dilute limit (Section II.B) and the size effect on the thermal conductivity of the matrix and the coating, due to the small sizes of the coating and the CNFs (Section II.C).

The modeling of the composite thermal conductivity is done by assuming that: 1) the aspect ratio (length/diameter) of the fibers is small enough to warrantee their randomness inside the matrix and in the absence of percolation, at least within a wide range of volume fractions of the fibers. This could be easily achieved for aspect ratio on the order of or smaller than 100. 2) The aspect ratio of the fibers is big enough (much greater than the unity) to make sure that they can provide a preferential direction of conduction along their axis. The lower bound of this aspect ratio can be accurately determined through the geometrical factors, which define the geometry effect of particles [16]. Figure 2 shows these factors along the major $\left(L_{\|}\right)$and minor $\left(L_{\perp}\right)$ axes of an ellipsoidal particle. Note that as the aspect ratio of the ellipsoid increases, the geometrical factors $\left(L_{\|}, L_{\perp}\right)=(1 / 2,0)$, which are the values for a very long cylindrical fiber of circular cross section. For an aspect ratio of $a_{\|} / a_{\perp}=10$, the geometrical factors deviate from their corresponding values for an infinite fiber by just about $2 \%$, and this deviation reduces for higher 
aspect ratios. This indicates that a fiber with an aspect radio equal or greater than 10 has a very similar geometrical effect than another with an infinite aspect ratio. Thus, it is clear that our assumptions for the composite under consideration can be fulfilled when the aspect ratio of the fibers is greater than 10 but smaller than 100 .

\section{A. Tri-Phase Thermal Conductivity Model under the Dilute Limit}

The description of the effective thermal conductivity of a tri-phase composite shown in Fig. (a) requires capturing the effects of the thermal and geometrical properties of the CNFs, its metallic coating, and the dielectric matrix. Based on the temperature profile inside a composite exposed to a constant heat flux and assuming that the volume fraction $f$ of coated spheroidal particles is small enough ( $f<<1$, dilute limit) to neglect their interactions, Ordonez-Miranda et al.[11] have derived a model for the effective thermal conductivity $k$ of a tri-phase composite. For cylindrical particles with random and aligned spatial distributions, as is the case of CNFs shown in Fig. 1(a), Ordonez-miranda et al. results reduces to [11]

$$
\frac{k}{k_{m}}=\frac{3+\left(\beta_{1}+\beta_{3}\right) f}{3-\beta_{1} f},
$$

$$
\frac{k_{e \perp}}{k_{m}}=\frac{2+\beta_{1} f}{2-\beta_{1} f},
$$

$$
\frac{k_{e \|}}{k_{m}}=1+\beta_{3} f
$$

\section{being $k_{e \perp}$ and $k_{e \|}$ the effective thermal conductivities in the directions perpendicular and parallel}

to the axis of the aligned wires, respectively; and

$$
\beta_{1}=2 \frac{1-a_{k} /(a+\delta)-k_{m} / k_{1}}{1+a_{k} /(a+\delta)+k_{m} / k_{1}}
$$




$$
\begin{aligned}
& \beta_{3}=k_{3} / k_{m}-1, \\
& \frac{k_{1}}{k_{c}}=\frac{2+\alpha_{1} v}{2-\alpha_{1} v}, \quad \frac{k_{3}}{k_{c}}=1+\alpha_{3} v, \\
& \alpha_{1}=2 \frac{k_{\perp}-k_{c}}{k_{\perp}+k_{c}}, \quad \alpha_{3}=\frac{k_{\|}}{k_{c}}-1,
\end{aligned}
$$

where $a_{k}=R k_{m}$ is the Kapitza radius at the coating-matrix interface, $R$ is the corresponding interfacial thermal resistance, and $v=a^{2} /(a+\delta)^{2}$ is the volume fraction of the CNFs relative to their total volume with coating of thickness $\delta$ (see Fig 1(b)). Note that Eqs. (1) depend on the axial $\left(k_{\|}\right)$and radial $\left(k_{\perp}\right)$ thermal conductivities of the CNFs, such that the coating effect vanishes for $k_{\|}=k_{\perp}=k_{c}$. Within the metallic coating, the heat conduction is determined by the electron-electron, phonon-phonon, and electron-phonon scattering mechanisms, as shown in Fig. 1(b). The effect of electron-phonon coupling is expected to increases as $\delta$ is scaled down to values comparable or smaller than the electron and phonon mean free paths, due to the lack of equilibrium between the electron and phonon gases at these scales. In this case, the effect of the electron-phonon interactions within the metallic coating can be approximately described through the electron-phonon coupling factor $G,[11]$ which is defined in the two-temperature model of heat conduction.[17, 18] According to this model, the thermal conductivity $k_{c}$ of the coating is given by [11]

$$
\begin{gathered}
k_{c}=\frac{k_{e}+k_{p}}{\chi} \\
\chi=1+\frac{k_{e}}{k_{p}} \frac{d}{a} \frac{i_{1}(a / d)}{i_{1}^{\prime}(a / d)},
\end{gathered}
$$




$$
d=\sqrt{\frac{k_{e} k_{p}}{G\left(k_{e}+k_{p}\right)}},
$$

where $d$ is the electron-phonon coupling length, $i_{1}$ the modified spherical Bessel function of the

first kind and order 1 , and the prime (') indicates derivative of $i_{1}$ with respect to its argument.

Given that $\chi>1$, Eq.(3a) introduces a reduction to the thermal conductivity $k_{e}+k_{p}$ of the coating and therefore on the composite thermal conductivity, due to the electron-phonon coupling. For common metals such as copper, silver, and others at room temperature; $d \approx 100 \mathrm{~nm}[17]$, which indicates that for nanoparticles with radius $a<<$, the factor $\chi \rightarrow 1+k_{e} / k_{p}$

Equation (1) along with Eqs. (2) and (3) generalize many previous models reported in the literature under the dilute limit $(f<<1)$, in which the interactions among CNFs are negligible[19-21]. For higher concentrations of CNFs, however, the fiber-fiber interactions need to be considered. Furthermore, when the size (thickness) of the CNFs (coating) is comparable to or smaller than the phonon (electron and/or phonon) MFP inside the dielectric matrix (metallic coating), the thermal conductivity of these components has to be adjusted in Eq. (1) to take into account the size effects $[22,23]$. The effect of fiber-fiber interactions under the non-dilute limit and the size effect on the matrix are going to be addressed in what follows.

\section{B. Effect of Fiber-Fiber Interaction}

At high concentrations of CNFs, the heat transport through a CNF is strongly affected by the presence of its neighbors. Based on this fact, Ordonez-Miranda et al.[24] have recently shown that the effect of the fiber-fiber interactions on the thermal conductivity of composites can be described by means of a crowding factor defined as the effective volume fraction of the CNFs. 
This crowding factor model establishes that the effective thermal conductivity of a composite made up of particles with a volume fraction $f$ up to their maximum packing fraction $f_{0}$ $\left(f \leq f_{0}\right)$, is given by $k=k_{m} \exp (C f /(1-\gamma \psi f))$, where $C$ and $\gamma$ are constants, and

$$
\psi=1+\frac{\left(1-f_{0}\right) f}{f_{0}^{2}} .
$$

Equation (4) shows that the effect of $f_{0}$ on the thermal conductivity of the composite appears for high concentrations $\left(f \rightarrow f_{0}\right)$, and it becomes negligible for $f<<f_{0}$ (dilute limit). For randomly oriented cylindrical particles, as is the case of the CNFs in Fig. 1(a), the maximum packing fraction is $f_{0}=52 \%[25]$. In the dilute limit, the first-order approximation of the crowding factor model reduces to $k=k_{m}(1+(C-\gamma) f) /(1-\gamma f)$, which by comparison with Eqs. (1a) and (1b) yields $3 \gamma=\beta_{1}$ and $3 C=2 \beta_{1}+\beta_{3}$, for fibers with random distribution, and $2 \gamma=\beta_{1}$ and $C=\beta_{1}$, for aligned fibers. The composite thermal conductivity for any volume fraction within the interval $f \leq f_{0}$ is then determined by

$$
\begin{aligned}
& \frac{k}{k_{m}}=\exp \left(\frac{\left(2 \beta_{1}+\beta_{3}\right) f}{3-\beta_{1} \psi f}\right), \\
& \frac{k_{e \perp}}{k_{m}}=\exp \left(\frac{2 \beta_{1} f}{2-\beta_{1} \psi f}\right),
\end{aligned}
$$

and Eq. (1c) does not change due to the absence of crowding of fibers along their axial direction [24]. Equations (5a) and (5b) extends not only the range of application of Eqs. (1a) and (1b) to high CNF concentrations, but also generalizes many models reported in the literature, from the pioneer work of Maxwell[26] to recent works by Nielsen [25] and Nan et al.[19] Taking into 
account that $\beta_{1}<1$ (Eq. 2a)), Eq. (5a) establishes that the composite thermal conductivity is greater than the one of the matrix $\left(k>k_{m}\right)$ for $2 \beta_{1}+\beta_{3}>0$. Considering that the axial thermal conductivity of the CNFs is greater than that of the matrix $\left(k_{3}>k_{m}\right)$ and according to Eqs. (2a) and (2b), this condition is satisfied when

$$
\frac{k_{m}}{k_{1}}+\frac{a_{k}}{a+\delta}<1
$$

which is the condition for the enhancement of the thermal conductivity of the matrix $\left(k>k_{m}\right)$, and depends on the fiber radius, matrix-coating Kapitza radius, and the size effects through the thermal conductivity of the matrix $k_{m}$, as shown in Eq. (7a) below. In this way, Eqs. (6) represents the "golden rule" for the design and fabrication of composites with thermal conductivities higher than the one of their matrix. If Eq. (6) is not fulfilled, the composite thermal conductivity will be smaller than the one of the matrix $\left(k<k_{m}\right)$, which could happen when the interface thermal resistance between the coating and matrix is large enough to satisfy the condition $a_{k} \gg>a+\delta$.

\section{Size Effects on the Effective Thermal Conductivity}

Due to the relatively small size of the CNFs, their thermal conductivity in both the axial $\left(k_{\|}\right)$ and radial $\left(k_{\perp}\right)$ directions could be size-dependent. These thermal conductivities are often reported as effective measured values, which should be directly used as the inputs for Eq. (2c). The size effects of the metallic coating on the thermal conductivities of the coating and matrix, are also taken into account as follows: 
- As a result of the phonon-phonon and phonon-coating scattering processes within the matrix, the (phonon) thermal conductivity $k_{m}$ of the dielectric matrix is reduced with respect to its bulk values $K_{m}$, according to [22, 23]

$$
k_{m}=\frac{K_{m}}{1+2 l_{m} f / \pi(a+\delta)},
$$

where $l_{m}$ is the mean free path (MFP) of phonons in the matrix.

- Similarly, the interfacial scattering of the electrons and phonons inside the metallic coating at the coating-matrix interface alters the effective thermal conductivity of both the electronic $\left(k_{e}\right)$ and phononic $\left(k_{p}\right)$ components. Such effective thermal conductivities can be written in parallel as reduced bulk electron $\left(K_{e}\right)$ and phonon $\left(K_{p}\right)$ thermal conductivities, [22, 23]

$$
k_{j}=\frac{K_{j}}{1+l_{j} / L},
$$

where $l_{j}$ for $j=e(j=p)$ is the intrinsic electron (phonon) MFP of the metallic coating material and $L$ is the average distance traveled by these energy carriers due to the scattering with the boundaries of the coating. Equations (7a) and (7b) have been derived by assuming that the intrinsic carrier scattering and the carrier-boundary scattering are independent with each other, and therefore the Matthiessen's rule is valid.

As shown in Fig. 1(b), the energy carriers may undergo multiple reflections at the inner ( $r=a)$ and outer $(r=a+\delta)$ surfaces of the coating. The average value of $L$ can then be defined as follows

$$
L=\frac{a \bar{L}_{i}+(a+\delta) \bar{L}_{o}}{2 a+\delta},
$$


where $\bar{L}_{i}$ and $\bar{L}_{o}$ are the mean distances travelled by an energy carrier reflected from the inner and outer surfaces of the coating, respectively. To calculate these two distances, the energy carriers are assumed to undergo diffusive scattering at the interfaces of the coating, i.e, the electrons and phonons are reflected from the boundary surfaces with equal probability to any direction. Let $L_{i}$ be the modulus of a vector connecting two arbitrary points from the inner to the outer surfaces of the cylindrical coating, then according to the geometry considerations shown in Fig. 3(a), its projection over the cross section (plane $x y$ ) of the coating is given by

$$
L_{i} \sin \theta=\sqrt{a^{2}+b^{2}-2 a b \cos \phi},
$$

where $b=a+\delta$ and $\theta$ is the usual polar angle of spherical coordinates, between the axis of the tube and the vector of modulus $L_{i}$. The average of this length over all possible directions is determined by its weighted average over the differential of solid angle $d \Omega=\sin \theta d \theta d \phi$, as follows

$$
\bar{L}_{i}=\frac{1}{\phi_{i}} \int_{0}^{\phi_{i}} d \phi \int_{0}^{\pi / 2} L_{i} \sin \theta d \theta
$$

where $\cos \phi_{i}=a / b$ with $\phi_{i}<\pi / 2$ (see Fig. 3(a)), and the range of integration over the polar angle has been reduced to $0 \leq \theta \leq \pi / 2$, because of the symmetry of the problem. By combining Eqs. (9) and (10), one finds

$$
\bar{L}_{i}=\frac{\pi(a+b)}{\phi_{i}}\left[E\left(\frac{\pi}{2}, \xi\right)-E\left(\frac{\pi-\phi_{i}}{2}, \xi\right)\right],
$$

where $\xi=2 \sqrt{a b} /(a+b)$ and $E($,$) is the incomplete elliptic integral of second kind [27].$ 
By applying the law of cosines and basic trigonometry, Fig. 3(b) shows that the modulus $L_{o}$ of a vector connecting two arbitrary points from the outer to the inner surfaces of the cylindrical coating is determined by

$$
L_{o} \sin \theta=\left\{\begin{array}{ll}
b \cos \phi-\sqrt{a^{2}-b^{2} \sin ^{2} \phi}, & \phi \leq \phi_{o} \\
2 b \cos \phi & , \quad \phi>\phi_{o}
\end{array},\right.
$$

where $\sin \phi_{o}=a / b$ with $\phi_{0}<\pi / 2$. Similarly to the previous case, the average of $L_{o}$ over all possible directions is found to be

$$
\bar{L}_{o}=2 b-a-a E\left(\phi_{0}, b / a\right) \text {. }
$$

Finally, the exact value of the carrier-boundary mean free path $L$ is obtained by substituting Eqs. (11) and (13) into Eq. (8). Based on the asymptotic values of the involved elliptic integrals for a thin coating $(\delta<<b)$ [27], Eq. (8) reduces to

$$
L \approx \frac{\delta}{\pi}[2(1+\ln 2)+\ln (1+2 a / \delta)]
$$

Equation (14) indeed represents an accurate estimation of the exact value of $L$, for a wide range of the ratio $\delta / a$. The deviation on $L$ from its exact value is just about $5 \%$ for $\delta / a=1$, and it takes lower values for smaller ratios $\delta / a$.

Equation (5) along with Eqs. (2)-(4), (7), (8) and (14) represent our model for describing the effective thermal conductivity of composites made up a dielectric matrix embedded with metallic-coated CNFs. Our results take into account the anisotropy of the thermal conductivity of the CNFs (Eqs. (2a)-(2d)), the thermal resistance of the coating-matrix interface (Eq. (2a) and (2b)), the electron-phonon interactions inside the metallic coating (Eq. (3)), the interaction between fibers(Eq. (4)), and the size effects of the coating (Eqs. (7), (8), and (14)); and therefore it is expected to provide an accurate description of the thermal conductivity of the composites 
shown in Fig. 1(a). In absence of coating $(\delta=0)$, Eqs. (2c) and (2d) reduces to $k_{1}=k_{\perp}$ and $k_{3}=k_{\|}$and therefore Eqs. (2a) and (2b) take the form

$$
\begin{gathered}
\beta_{1}=2 \frac{1-a_{k} / a-k_{m} / k_{\perp}}{1+a_{k} / a+k_{m} / k_{\perp}}, \\
\beta_{3}=k_{\|} / k_{m}-1,
\end{gathered}
$$

where the effective thermal conductivity $k_{m}$ of the matrix is given by Eq. (7a). It is therefore clear that for $\delta=0$, the effective thermal conductivity of the composite is still given by Eq. (5), with the parameters $\beta_{1}$ and $\beta_{2}$ defined by Eqs. (15a) and (15b).

\section{APPLICATIONS AND DISCUSSIONS}

In this section, we present the comparison of the effective thermal conductivity of CNFpolymer nanocomposite with and without a metallic coating. Based on the input data summarized in table I, the effect of different metallic coatings is analyzed as a function of their thickness. We note here that the effective thermal conductivity of CNFs reported as experimental data can be different for different sizes due to the size effects of phonon transport. However, for simplicity and without losing the generality, we use an explicit and constant value when changing the size of the CNFs.

Figures 4(a) and 4(b) show the normalized thermal conductivity of a composite as a function of the volume fraction of CNFs coated with a copper layer and embedded in a polyester resin, for different radii and coating thicknesses, respectively. Given that the thermal conductivity of the CNFs and their metallic coating is much higher than the one of the matrix, the effective thermal conductivity of the composites $k$ increases not only with the volume fractions of the coated CNFs but also with the radius of the CNFs and the thickness of their coating. The comparison of Figs. 4(a) and 4(b) shows that the effective thermal conductivity $k$ is more sensitive to the 
changes of the CNFs radius than to the thickness of their metallic coating. This is due to the strong effect of electron-phonon coupling that reduces the effective thermal conductivity of the coating, which indicates that the enhancement of the effective thermal conductivity of the composites can been better maximized by increasing the radius of the fibers instead of widening the relatively expensive metallic coating. For the volume fraction $f=30 \%$ of CNFs with $(a, \delta)=(50,10) \mathrm{nm}$, Fig. 4(a) shows that the enhancement of the thermal conductivity of the polymeric matrix is about $32 \%$, which is similar to the increase of $31 \%$ for $(a, \delta)=(20,50) \mathrm{nm}$ and the same volume fraction, as shown in Fig. 4(b). Keeping the volume fraction fixed at $f=30 \%$, however, this enhancement is just about $5 \%$ when the thickness of the coating widens from $\delta=10 \mathrm{~nm}$ to $\delta=50 \mathrm{~nm}$, which is small compared with the corresponding increase of $13 \%$, when the fiber radius increases from $a=10 \mathrm{~nm}$ to $a=50 \mathrm{~nm}$.

The normalized thermal conductivity of a polyester matrix composite as a function of the volume fraction of CNFs coated with different metallic is shown in Fig. 5. The composites made up of CNFs coated with metallic layers have a thermal conductivity $k$ much larger than the corresponding one for uncoated CNFs. At the volume fraction $f=30 \%$, this increase is about $27 \%$ for the case of copper, and it takes higher values when the concentration of CNFs increases. This extraordinary enhancement generated by the metallic coating is due to its high thermal conductivity and the predominance of the surface effects over the volumetric ones at nanoscales.

Note that the curves of $k$ for the coatings of silver and copper, and nickel and indium are almost overlapped, which is because of the similar bulk thermal conductivities for each pair of these materials, as reported in Table I. Furthermore, by changing the metallic coating from silver or copper to nickel or indium, the composite thermal conductivity does not change remarkably in the whole range of volume fractions of CNFs. For instance, at $f=30 \%$, the increase on the 
composite thermal conductivity is just about $2 \%$ when the thermal conductivity of the coating changes $453 \%$, from indium to silver. This weak sensitivity of $k$ to the thermal conductivity of the metallic coating is consistent with the relatively small variation of $k$ with the coating thickness (Fig. 4(b)), and it is due to the reduction of the effective electron and phonon thermal conductivity of the metallic coating as a result of the strong electron-phonon interactions at nanoscales, as established by the parameter $\chi$ in Eq. (3b). For a $50 \mathrm{~nm}$-thick coating of silver, $\chi \approx 40$, which reduces its bulk thermal conductivity of from $430 \mathrm{~W} / \mathrm{m} . \mathrm{K}$ to $10.8 \mathrm{~W} / \mathrm{m} . \mathrm{K}$, that is still two orders of magnitude larger than the one of the matrix $\left(K_{m}=0.3 \mathrm{~W} / \mathrm{m} . \mathrm{K}\right)$. It is therefore clear that expensive metallic coatings could be replaced by other much cheaper, without reducing significantly the enhancement of the thermal conductivity of the composite. Furthermore, Fig. 5(b) shows that this enhancement reduces as the thermal conductivity of the matrix increases, such that values high enough $\left(K_{m}=10 \mathrm{~W} / \mathrm{m} . \mathrm{K}\right)$, the composite thermal conductivity can even decreases as the volume fraction of the CNFs increases. This is due to the increasing effect of the interface thermal resistance, through the Kapitza radius $\left(a_{k}=R k_{m}\right)$, which is proportional to the thermal conductivity of the matrix and leads to the nonfulfillment of the "golden rule" (Eq. (6)) for the enhancement of the composite thermal conductivity. To fulfill Eq. (6) and therefore to optimize the enhancement of the thermal conductivity of the matrix with coated CNFs, their total radius and radial thermal conductivity should be greater than the Kapitza radius $\left(a+\delta>a_{k}\right)$ and the effective thermal conductivity of the matrix $\left(k_{1}>k_{m}\right)$, respectively.

The experimental data [28] for the thermal conductivity of a composite made up of aligned carbon fibers with a copper coating and embedded in a epoxy matrix are shown in Fig. 6, as a function of the volume fraction of the coating and in comparison with the theoretical predictions 
of Eqs. (1c) and (5b). Given that the volume fractions of the fibers $\left(f_{p}\right)$ and their coating $\left(f_{c}\right)$ are related by $f_{c} / f_{p}=(b / a)^{2}-1$, the volume fraction $(f)$ of the coated fibers is given by $f=f_{p}+f_{c}=f_{c} /\left(1-(a / b)^{2}\right)$. Note that the thermal conductivities along the directions parallel and perpendicular to the axis of the aligned fibers increase with copper content, and they are both in fairly good agreement with the predictions of the proposed theoretical model. The relatively small difference between the experimental and theoretical results is reasonable due to the fact that the model assumes that the fibers are uni-directionally aligned, while in the prepared samples they were slight twisted [28]. The composite with $12 \%$ of copper content has an alongfibers axis thermal conductivity of $47.2 \mathrm{~W} / \mathrm{m} . \mathrm{K}$, which is 12 times larger than that perpendicular to the axis of the fibers. This is expected due to two facts: (i) the thermal conductivity of the carbon fibers along their axis is one order of magnitude larger than that in the perpendicular direction $\left(k_{\|}, k_{\perp}\right)=(8.04,0.84) \mathrm{W} / \mathrm{m} \cdot \mathrm{K}$, and (ii) the fibers and their coating represent paths of heat conduction along the fibers axis, which enhances the overall thermal conductivity of the composite in that direction. It is therefore clear that the thermal conductivity of these composites can be enhanced not only by increasing the content of the metallic coating of the fibers, but also by tuning their alignment.

\section{CONCLUSIONS}

The thermal conductivity of composites made up of randomly oriented and aligned carbon nanofibers coated with a metallic layer and embedded in a dielectric matrix has been modeled and analyzed, by taking into account the coating-size effects and the different scattering processes of the energy carriers inside the coating and matrix. It has been shown that: 1) the metallic coating has an extraordinary effect on the enhancement of the composite thermal 
conductivity with respect to its values for uncoated fibers. For a volume fraction of $30 \%$ of fibers coated with copper, this increase is as high as $27 \%$, which increases significantly with the radius of the fiber, their volume fraction, and the thickness of the coating. 2) A remarkable change on the thermal conductivity of the coating of $453 \%$, between indium and silver, modified the thermal conductivity of the composite by just $2 \%$, for a concentration of coated fibers of $30 \% .3$ ) The enhancement of the composite thermal conductivity occurs when the radius and radial thermal conductivity of the fibers are greater than the matrix-coating Kapitza radius and the effective thermal conductivity of the matrix, respectively. The predictions of the proposed model are in fairly good agreement with experimental data reported in the literature for aligned carbon fibers and they can be useful to optimize the thermal performance of new cost-efficient composite materials. 


\section{References}

[1] Stankovich S, Dikin DA, Dommett GHB, Kohlhaas KM, Zimney EJ, Stach EA, et al. Graphene-based composite materials. Nature 2006;442:282-286.

[2] Balandin AA. Thermal properties of graphene and nanostructured carbon materials. Nature Materials 2011;10:569-581.

[3] Thostenson ET, Li CY, Chou TW. Nanocomposites in context. Compos Sci Technol 2005;65:491-516.

[4] Reinecke BN, Shan JW, Suabedissen KK, Cherkasova AS. On the anisotropic thermal conductivity of magnetorheological suspensions. J Appl Phys 2008;104:023507.

[5] Coleman JN, Khan U, Blau WJ, Gun'ko YK. Small but strong: A review of the mechanical properties of carbon nanotube-polymer composites. Carbon 2006;44:1624-1652.

[6] Garcia EJ, Hart AJ, Wardle BL, Slocum AH. Fabrication and nanocompression testing of aligned carbon-nanotube-polymer nanocomposites. Adv Mater 2007;19:2151-2156.

[7] Ordonez-Miranda J, Alvarado-Gil JJ. Thermal conductivity of nanocomposites with high volume fractions of particles. Compos Sci Technol 2012;72:853-857.

[8] Lucio JL, Alvarado-Gil JJ, Zelaya-Angel O, Vargas H. On the Thermal-Properties of a TwoLayer System. Physica Status Solidi a-Applied Research 1995;150:695-704.

[9] Tam AC. Applications of photoacoustic sensing techniques. Rev Mod Phys 1986;58:381-431.

[10] Tominaga T, Ito K. Theory of photoacoustic measurements of the thermal diffusivity of two-layers samples. Jpn J Appl Phys 1988;27:2392-2397.

[11] Ordonez-Miranda J, Yang R, Alvarado-Gil JJ. A model for the effective thermal conductivity of metal-nonmetal particulate composites. J Appl Phys 2012;111:044319.

[12] Tian WX, Yang RG. Thermal conductivity modeling of compacted nanowire composites. J Appl Phys 2007;101:054320.

[13] Yang RG, Chen G, Dresselhaus MS. Thermal conductivity of simple and tubular nanowire composites in the longitudinal direction. Phys Rev B 2005;72:125418.

[14] Yang RG, Chen G, Dresselhaus MS. Thermal conductivity modeling of core-shell and tubular nanowires. Nano Lett 2005;5:1111-1115. 
[15] Yang RG, Chen G. Thermal conductivity modeling of periodic two-dimensional nanocomposites. Physical Review B 2004;69:195316.

[16] Kim G. Thermo-physical responses of polymeric composites tailored by electric field. Compos Sci Technol 2005;65:1728-1735.

[17] Ordonez-Miranda J, Yang RG, Alvarado-Gil JJ. The effect of the electron-phonon coupling on the effective thermal conductivity of metal-nonmetal multilayers. J Appl Phys 2011;109:094310-094316.

[18] Majumdar A, Reddy P. Role of electron-phonon coupling in thermal conductance of metalnonmetal interfaces. Appl Phys Lett 2004;84:4768-4770.

[19] Nan CW, Birringer R, Clarke DR, Gleiter H. Effective thermal conductivity of particulate composites with interfacial thermal resistance. J Appl Phys 1997;81:6692-6699.

[20] Milton GW. The Theory of Composites. Cambridge; New York: Cambridge University Press; 2002.

[21] Torquato S. Random Heterogeneous Materials. New York: Springer-Verlag; 2001.

[22] Ordonez-Miranda J, Yang RG, Alvarado-Gil JJ. On the thermal conductivity of particulate nanocomposites. Appl Phys Lett 2011;98:233111-233113.

[23] Minnich A, Chen G. Modified effective medium formulation for the thermal conductivity of nanocomposites. Appl Phys Lett 2007;91:073105-073107.

[24] Ordonez-Miranda J, Yang RG, Alvarado-Gil JJ. Crowding factor model for the thermal conductivity of particulate composites at non-dilute limit. J Appl Phys 2013;114:064306-064312. [25] Nielsen LE. The Thermal and Electrical Conductivity of Two-Phase Systems. Industrial \& Engineering Chemistry Fundamentals 1974;13:17-20.

[26] Maxwell JC. A treatise on electricity and magnetism. Oxford: Clarendon Press; 1873.

[27] Arfken GB, Weber HJ. Mathematical methods for physicists. 6th ed. Boston: Elsevier; 2005.

[28] Yu S, Park BI, Park C, Hong SM, Han TH, Koo CM. RTA-Treated Carbon Fiber/Copper Core/Shell Hybrid for Thermally Conductive Composites. Acs Appl Mater Inter 2014;6:74987503.

[29] Hasselman DPH, Donaldson KY, Liu J, Gauckler LJ, Ownby PD. Thermal-Conductivity of a Particulate-Diamond-Reinforced Cordierite Matrix Composite. J Am Ceram Soc 1994;77:1757-1760. 
[30] Lin Z, Zhigilei LV, Celli V. Electron-phonon coupling and electron heat capacity of metals under conditions of strong electron-phonon nonequilibrium. Physical Review B 2008;77.

[31] Hopkins PE, Kassebaum JL, Norris PM. Effects of electron scattering at metal-nonmetal interfaces on electron-phonon equilibration in gold films. J Appl Phys 2009;105:023710-023717. [32] Lewis TB, Nielsen LE. Dynamic Mechanical Properties of Particulate-Filled Composites. J Appl Polym Sci 1970;14:1449-1471.

[33] Small CG. Functional equations and how to solve them. New York, NY: Springer; 2007.

[34] Shenogin S, Gengler J, Roy A, Voevodin AA, Muratore C. Molecular dynamics studies of thermal boundary resistance at carbon-metal interfaces. Scripta Mater 2013;69:100-103.

Table I: Material properties at room temperature used in the calculations [18, 29-34].

\begin{tabular}{|c|c|c|c|c|c|c|}
\hline \multirow[t]{2}{*}{ Material } & \multicolumn{2}{|c|}{$\begin{array}{l}\text { Thermal conductivity } \\
\qquad(\mathrm{W} / \mathrm{m} . \mathrm{K})\end{array}$} & \multicolumn{2}{|c|}{$\begin{array}{l}\text { MFP } \\
(\mathrm{nm})\end{array}$} & \multirow[t]{2}{*}{$\begin{array}{l}\text { Coupling factor } \\
\left(10^{16} \mathrm{~W} / \mathrm{m}^{3} . \mathrm{K}\right)\end{array}$} & \multirow[t]{2}{*}{$\begin{array}{c}\mathrm{ITR}^{*} \\
\left(10^{-9} \mathrm{~m}^{2} \mathrm{~K} / \mathrm{W}\right)\end{array}$} \\
\hline & Electron & Phonon & Electron & Phonon & & \\
\hline $\mathrm{CNF}$ & --- & $\begin{array}{c}20-500(\|) \\
0.1(\perp)\end{array}$ & --- & 600 & --- & --- \\
\hline Silver & 420 & 10 & 4 & 6.5 & 2.5 & 3.6 \\
\hline Copper & 383 & 15 & 2.7 & 8.2 & 2.7 & 5.2 \\
\hline Nickel & 83 & 8 & 0.5 & 9.3 & 4.3 & 8.7 \\
\hline Indium & 76 & 6 & 0.38 & 7.1 & 5.8 & 9.4 \\
\hline $\begin{array}{l}\text { Polyester } \\
\text { resin }\end{array}$ & --- & 0.3 & --- & 5 & --- & --- \\
\hline
\end{tabular}




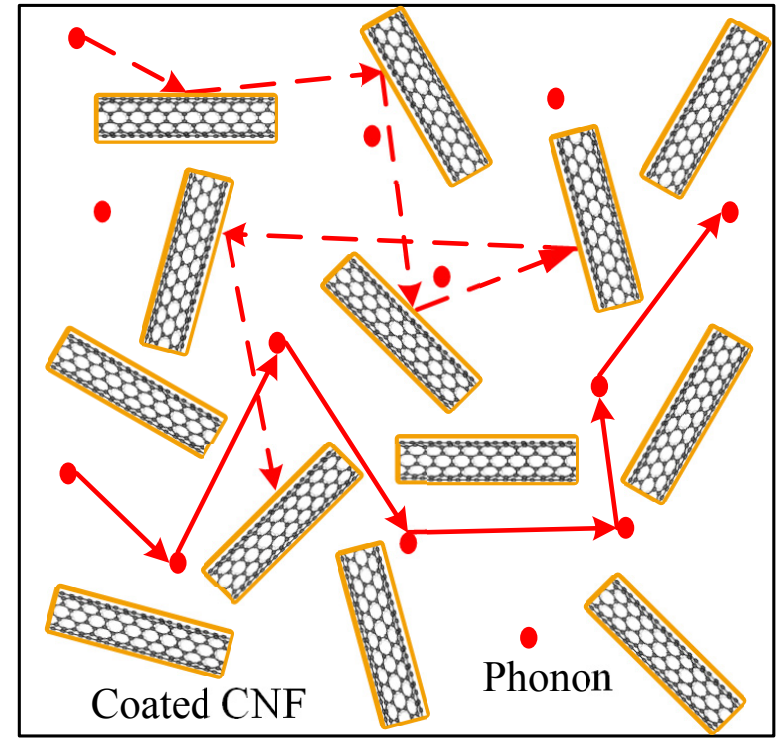

(a)

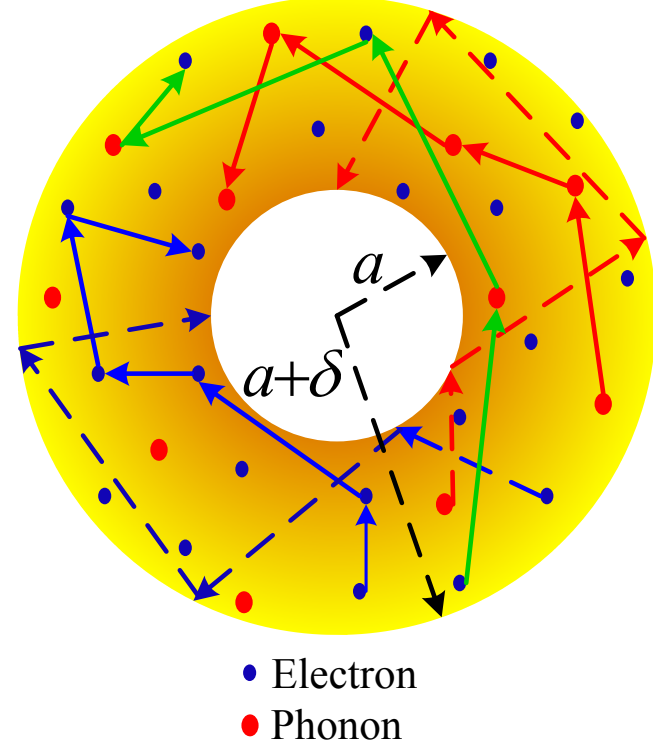

(b)

Fig. 1. (a) The phonon scattering processes inside the matrix, (b) the electron and phonon scattering processes in the $\delta$-thickness metallic coating of a CNF with radius $a$ (cross-section). 


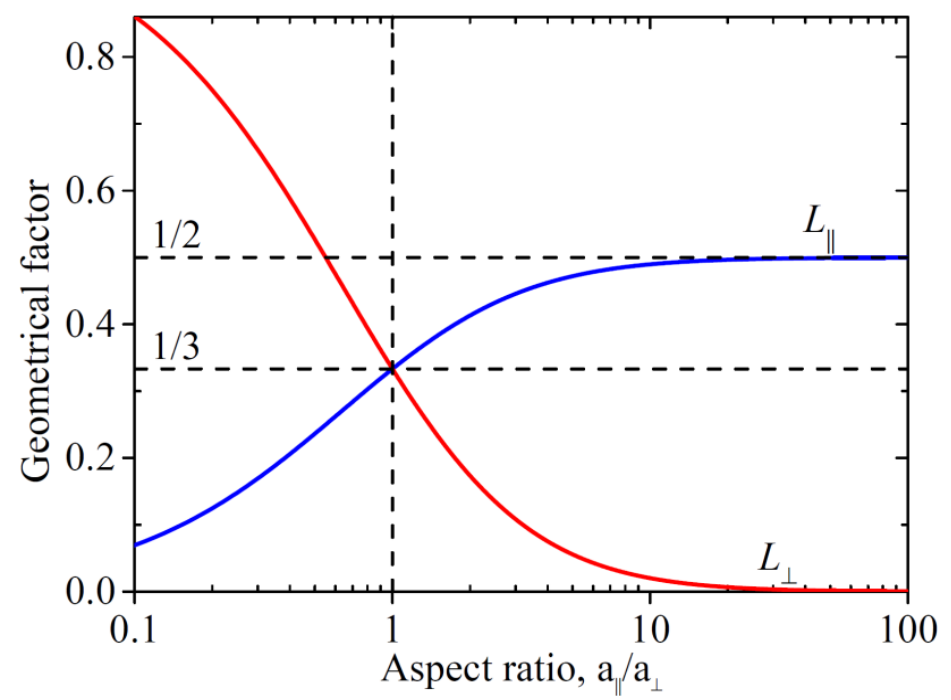

Fig. 2. Geometrical factors of an ellipsoidal particle as a function of its aspect ratio.

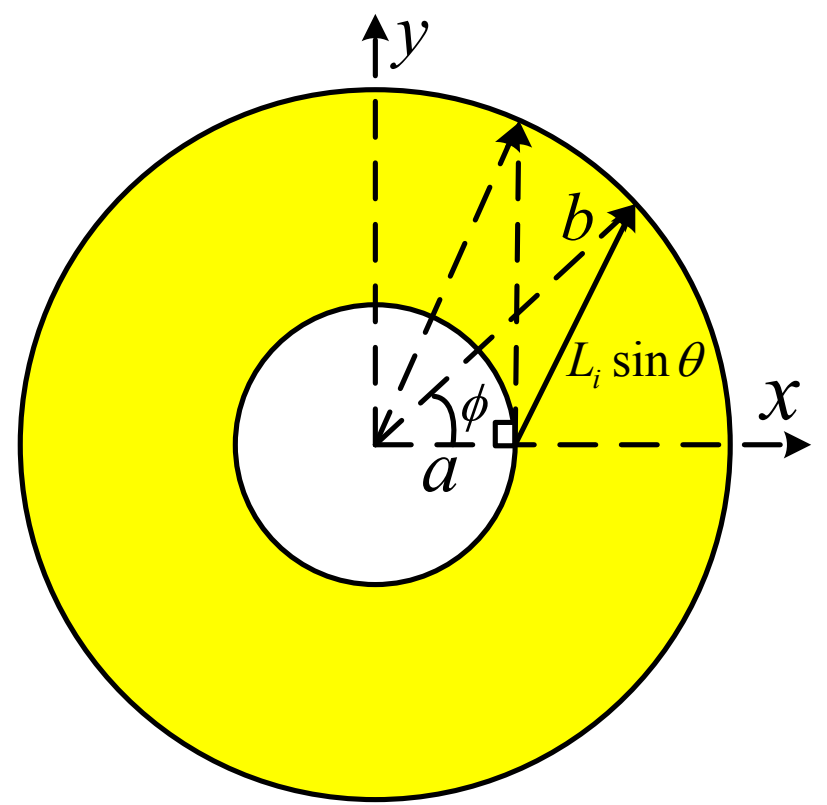

(a)

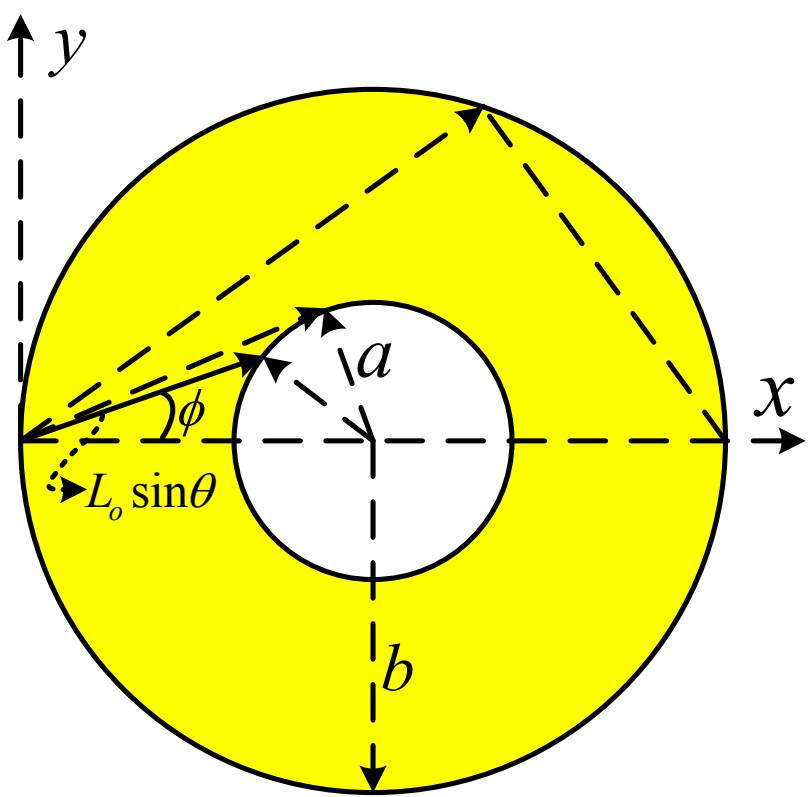

(b)

Figure 3. Cross section of the cylindrical coating and parameters to calculate the carrierboundary mean distance from the (a) inner and (b) outer interfaces. 


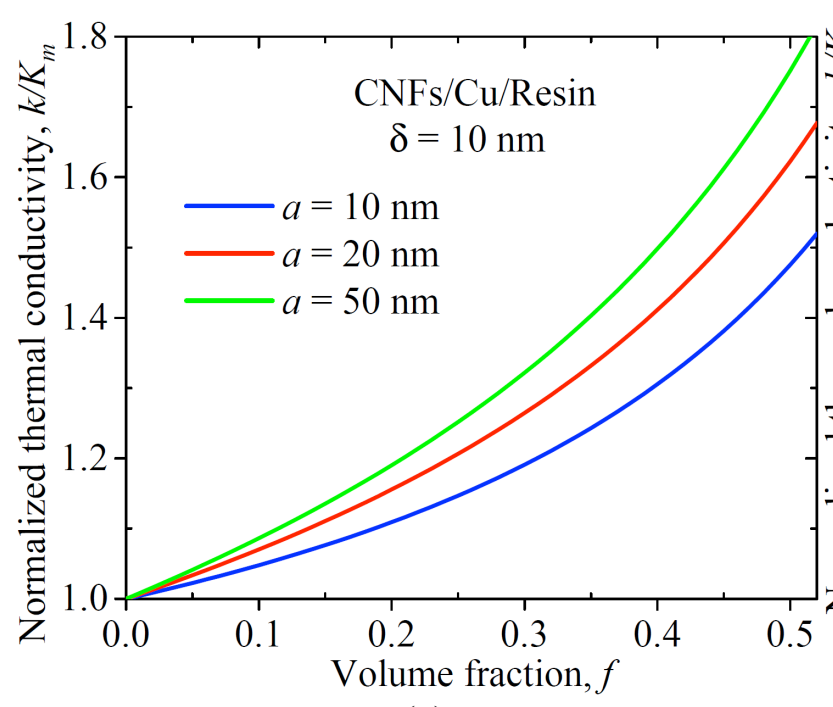

(a)

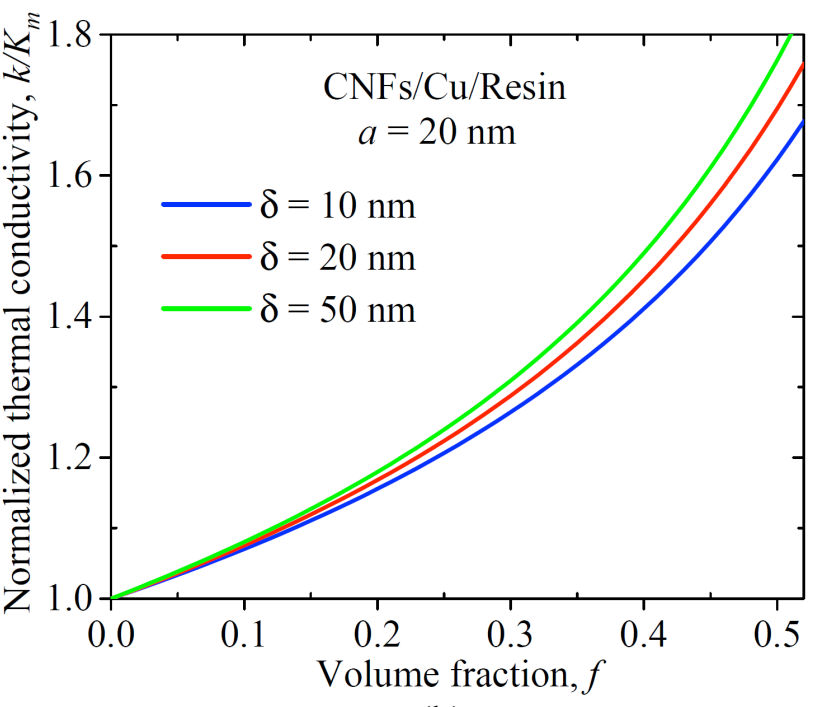

(b)

Figure 4. Normalized thermal conductivity of a composite as a function of the volume fraction of CNFs coated with a copper layer and embedded in a polyester resin, for three different (a) radii and (b) coating thicknesses. Calculations were performed with $k_{\|}=20 \mathrm{~W} / \mathrm{m} . \mathrm{K}$ and the data in Table I.

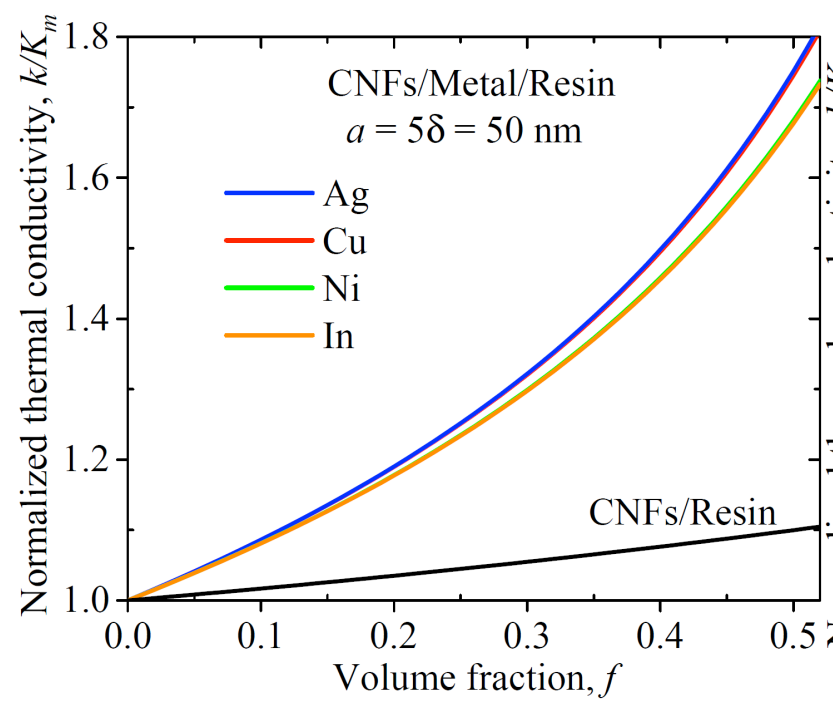

(a)

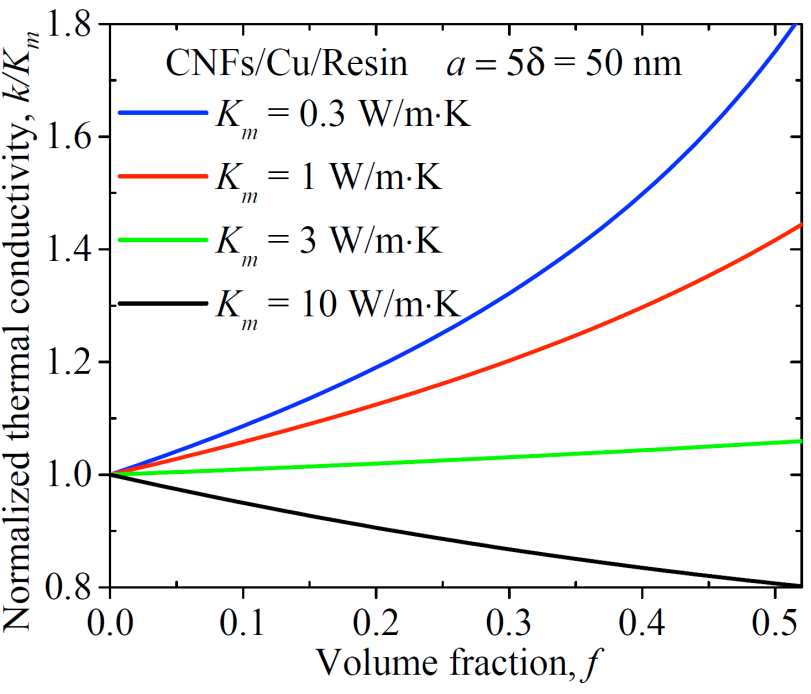

(b)

Figure 5: Normalized thermal conductivity of a composite as a function of the volume fraction of CNFs coated with (a) different metallic materials and embedded in a polyester resin, and (b) a copper layer and embedded in polyester resins of different thermal conductivities. Calculations were performed with $k_{\|}=20 \mathrm{~W} / \mathrm{m} . \mathrm{K}$ and with the data in Table I. 


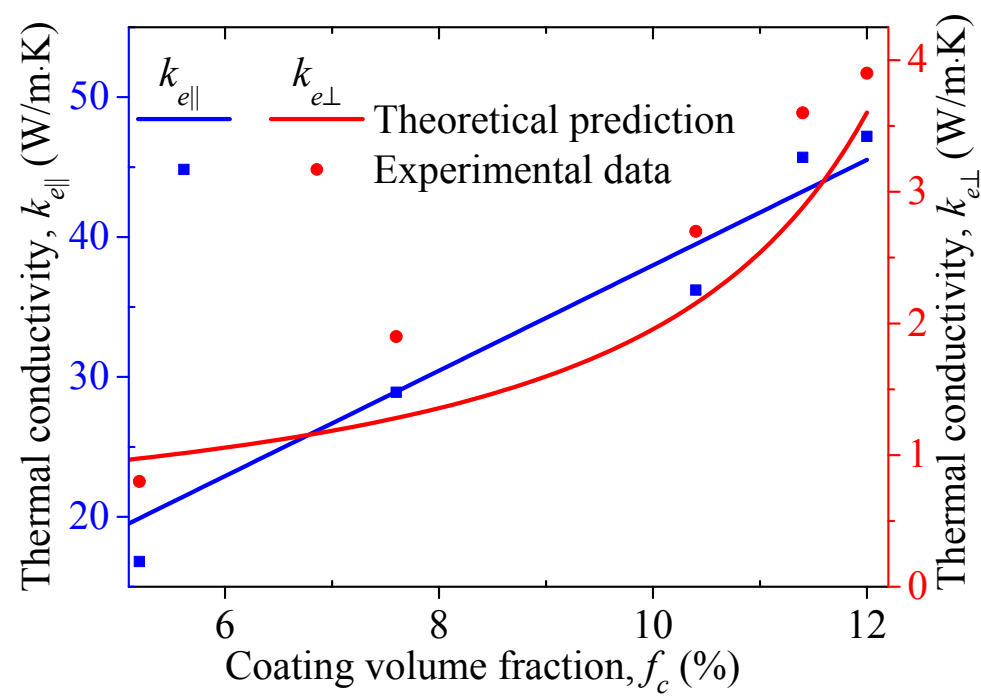

Figure 6: Thermal conductivity of a composite made up of aligned carbon fibers coated with copper and embedded in an epoxy matrix (bisphenol A diglycidyl ether), as a function of the volume fraction of the coating. Calculations were performed with $\left(k_{\|}, k_{\perp}\right)=(8.04,0.84) \mathrm{W} / \mathrm{m} \cdot \mathrm{K}$ and the experimental data reported by $\mathrm{Yu}$ et al.[28] 